\title{
THE SQUARE OF EVERY NONSEPARABLE GRAPH IS HAMILTONIAN
}

\author{
BY HERBERT FLEISCHNER
}

Communicated by Victor Klee, April 22, 1971

In June, 1963, at the Graph Theory Symposium in Smolenice, Czechoslovakia, M. Sekanina asked for the structure of those graphs whose square is Hamiltonian [1]. At the Graph Theory Colloquium in Tihany, Hungary, in September, 1966, C. St. J. A. Nash-Williams asked if it is true that the square of every nonseparable graph is Hamiltonian [2]. He noted there that L. W. Beineke and M. D. Plummer had also independently thought of this conjecture. Since that time this conjecture has become known by the names of Plummer and Nash-Williams.

The proof of this conjecture is divided in to four papers:

[a] On a certain spanning subgraph of a connected bridgeless graph.

[b] The total graph of a block is Hamiltonian.

[c] On line-critical blocks.

[d] The square of every nonseparable graph is Hamiltonian.

In $[\mathrm{a}]$ the following theorems are established:

Theorem A (TheOREM 1 IN [a]). Let $G$ be a connected bridgeless graph. Then there exists a connected spanning subgraph $S$ of $G$ with the following properties:

(1) $S=E \cup P$, where

(a) $E$ is a (not necessarily connected) Eulerian graph,

(b) $P$ is a (possibly empty) set of paths $p_{i}$ each two of which have at most one point in common, and any such point is an endpoint of both paths and belongs to $E$.

(2) Every point of $E$ is a point of at most two paths of $P$, and if it is in a path of $P$, then it is an endpoint of the path.

(3) Given two different points $v$ and $w, S=E \cup P$ can be constructed such that $\{v, w\} \subset V(E), v \notin V(P)=\bigcup_{i} V\left(p_{i}\right)$, and $w$ is incident to at most one path of $P$.

Theorem B (Theorem 3 in [a]). Let $G$ be a connected bridgeless graph. Then there exists a connected spanning subgraph $S$ of $G$ with the

AMS 1970 subject classifications. Primary 05C99.

Key words and phrases. Hamiltonian cycles, the square of a graph, Plummer-NashWilliams conjecture, square-of-a-block-problem.

Copyright:_( American Mathematical Society 1971 
following properties:

(1) $S=E \cup J \cup P$, where

(a) $E$ is a (not necessarily connected) Eulerian graph,

(b) $J$ is a trail joining the points $v$ and $w$, and $E \cap J=\varnothing$.

(c) $P$ is a (possibly empty) set of paths $p_{i}$ each two of which have at most one point in common, and any such point is an endpoint in both paths and belongs to $E \cup J$.

(2) Every point of $E \cup J$ is a point of at most two paths of $P$, and if it is in a path of $P$, then it is an endpoint of the path.

(3) Given two different points $v$ and $w, S=E \cup J \cup P$ can be constructed such that $v$ and $w$ are the endpoints of $J, v \in V(P)$, and $w$ is incident to at most one path of $P$.

Theorem $\mathrm{B}$ is an immediate consequence of Theorem A.

Now let $G$ be a $D T$-graph, i.e. a graph having the property that each line is incident to a point of degree two. Applying Theorems A and $\mathrm{B}$ to $D T$-graphs we obtain the following results.

Theorem C (Theorem 2 IN [b]). Let $G$ be a connected, bridgeless $D T$-graph, and let $v, w$ be any two points of $G$ with $\operatorname{deg} v>2, \operatorname{deg} w>2$. Then there exists in $G^{2}$ a Hamiltonian cycle $H$ containing lines $e, f$ incident to $v$, and a line $g$ incident to w, with $\{e, f, g\} \subset L(G)(L(G)$ is the lineset of $G$ ).

TheOREM D (TheOREM $4 \mathrm{IN}[\mathrm{b}]$ ). Let $G$ be a graph satisfying the hypothesis of Theorem $\mathrm{C}$. Then there exists a Hamiltonian path HP of $G^{2}$ joining $v$ and $w$, and $H P$ contains a line $e$ of $G$ incident to $v$.

Furthermore, it is proven in [b] that the square of every connected, bridgeless $D T$-graph is Hamiltonian-connected. This proves and generalizes Kronk's conjecture [3] that the total graph $T(G)$ of a block is Hamiltonian, because the subdivision graph $S(G)$ of a block is a connected, bridgeless $D T$-graph and $T(G)$ is isomorphic to $(S(G))^{2}$.

In [c], the existence of a $D T$-subblock $B_{0}$ of a line-critical block is proven in

Theorem E (TheOREM 2 IN [c]). Let $G$ be a line-critical block with $|D(G)|>0$. Then there is a line $e_{0} \in D(G)$ such that $G\left(e_{0}\right)$ contains an endblock which is a DT-subgraph of $G(D(G)=\{(x, y) \in L(G) \mid \operatorname{deg} x$ $>2$, deg $\left.y>2\}, G\left(e_{0}\right)=G-e_{0}\right)$.

In [d], Theorems C, D, and E are used to prove the PlummerNash-Williams conjecture. The proof is indirect and proceeds as follows.

We let $G$ be a block such that $|D(G)|$ is minimal and whose square 
is non-Hamiltonian.

If $|D(G)|=0$, then $G$ is a $D T$-block, hence, by Theorem $\mathrm{C}, G^{2}$ is Hamiltonian.

If $|D(G)|>0$, then $G$ is a line-critical block. By Theorem $\mathrm{E}, G$ contains a certain $D T$-subblock $B_{0}$. Replacing $B_{0}$ with a path of length three we obtain a line-critical block $G_{1}$ with $\left|D\left(G_{1}\right)\right|<|D(G)|$. Therefore, $G_{1}^{2}$ is Hamiltonian. We consider in $G_{1}^{2}$ a Hamiltonian cycle $H_{1}$ which contains a maximum number of lines of $G_{1}$. By applying Theorems $\mathrm{C}$ and $\mathrm{D}$, we may extend $H_{1}$ to a Hamiltonian cycle of $G^{2}$.

\section{BIBLIOGRAPHY}

1. M. Sekanina, Problem No. 28, Theory of Graphs and its Applications, Academic Press, New York, 1964.

2. C. St. J. A. Nash-Williams, Problem No. 48, Theory of Graphs, Academic Press, New York, 1968.

3. H. V. Kronk, Is the square of every non-separable graph Hamiltonian? Amer. Math. Monthly 76 (1969), 1045-1046.

State University of New York, Binghamton, New York 13901 